\title{
A gastrointestinal stromal tumor in the stomach: usefulness of computed tomographic volumetry
}

\author{
Takahiro Hashiba ${ }^{1}$, Kenji Oda ${ }^{1}$, Keijt Koda ${ }^{1}$, Nobuhiro Takiguchi ${ }^{2}$, Kazuhiro Seike ${ }^{1}$, and Masaru Miyazaki ${ }^{1}$ \\ ${ }^{1}$ Department of General Surgery, Graduate School of Medicine, Chiba University, Inohana, Chuo-ku, Chiba 260-8670, Japan \\ ${ }^{2}$ Department of General Surgery, Chiba Cancer Center, Chiba, Japan
}

\begin{abstract}
We report herein the case of a 70-year-old man who was found to have a gastrointestinal stromal tumor (GIST) in the stomach following sigmoid colon resection. Preoperative gastroscopic and barium examinations revealed a submucosal tumor, measuring $10 \mathrm{~cm}$, on the upper part of the stomach. Using computed tomography (CT) images (i.e., computed tomographic volumetry) the doubling time of this tumor was calculated, accurately, as 3.3 months, which suggested a high growth rate and malignancy. A laparotomy and partial gastric resection were performed. Histologically, the tumor consisted of spindle-shaped cells with oval nuclei. In immunohistochemical studies, the tumor cells were positive with respect to c-kit, CD34, and vimentin, but negative with respect to smooth muscle actin and S-100 protein. There were 15-16 mitoses per 50 high-power fields (HPFs), and the Ki-67 antigen (MIB-1) index was $\mathbf{2 5 . 5} \%$ in the most active areas, which also indicated malignancy. The final pathological diagnosis of this tumor was malignant GIST. The patient was found to have hepatic metastasis 27 months after the surgery, and he subsequently received a hepatic subsegmentectomy. To our knowledge, there are very few reports concerning the growth rate of GISTs. Computed tomographic volumetry is useful for the follow-up of small or irregularly shaped gastric submucosal tumors, and for making decisions regarding surgical intervention.
\end{abstract}

Key words Gastrointestinal stromal tumor (GIST) - Stomach - Doubling time $\cdot$ Computed tomographic volumetry $\cdot \mathrm{c}$-kit

\section{Introduction}

Gastrointestinal stromal tumors (GISTs) are mesenchymal tumors of the digestive tract with various clinical and biological characteristics. The concept of what constitutes a GIST has been changing due to the advent

Offprint requests to: $\mathrm{K}$. Oda

Received: February 27, 2004 / Accepted: August 23, 2004 of immunohistological and molecular techniques [1,2]. Recently, it has been elucidated that GISTs have a mutation in either the c-kit gene or the platelet-derived growth factor receptor-alpha (PDGFRA) gene [2,3]. Several types of c-kit mutations have been reported [4-9]. Clinically, GIST malignancy has been determined by several factors, such as tumor size, ulceration, and necrosis, as well as the mitotic activity of the tumor cells [10-17]. Generally, preoperative assessment of GIST malignancy is not easy. Here, we report a case of malignant gastric GIST, and describe the usefulness of calculating the doubling time of tumors by using computed tomographic volumetry.

\section{Case report}

A 70-year-old man underwent sigmoid colon resection due to sigmoid colon carcinoma in September 1995, and follow-up examinations were conducted at our hospital. In June 2001, the patient complained of epigastric pain, and visited a nearby hospital. Endoscopic examination showed a gastric submucosal tumor, and he was referred to our hospital for further examination in July 2001.

The patient had undergone endoscopic resections for urinary bladder cancer twice, in 1989 and 1992. His younger brother also suffered from urinary bladder carcinoma.

On admission in July 2001, physical examination revealed a 10-cm sized mass, with tenderness in the upper left abdomen. An upper gastrointestinal barium study and gastric endoscopic examination indicated a large submucosal tumor, without obvious ulceration, in the upper part of the stomach (Figs. 1, 2). Endoscopic ultrasonogram showed an approximately $10-\mathrm{cm}$ hypoechoic lesion with a hyperechoic area.

In this patient, computed tomography (CT) images had been taken every year for follow-up in regard to his 


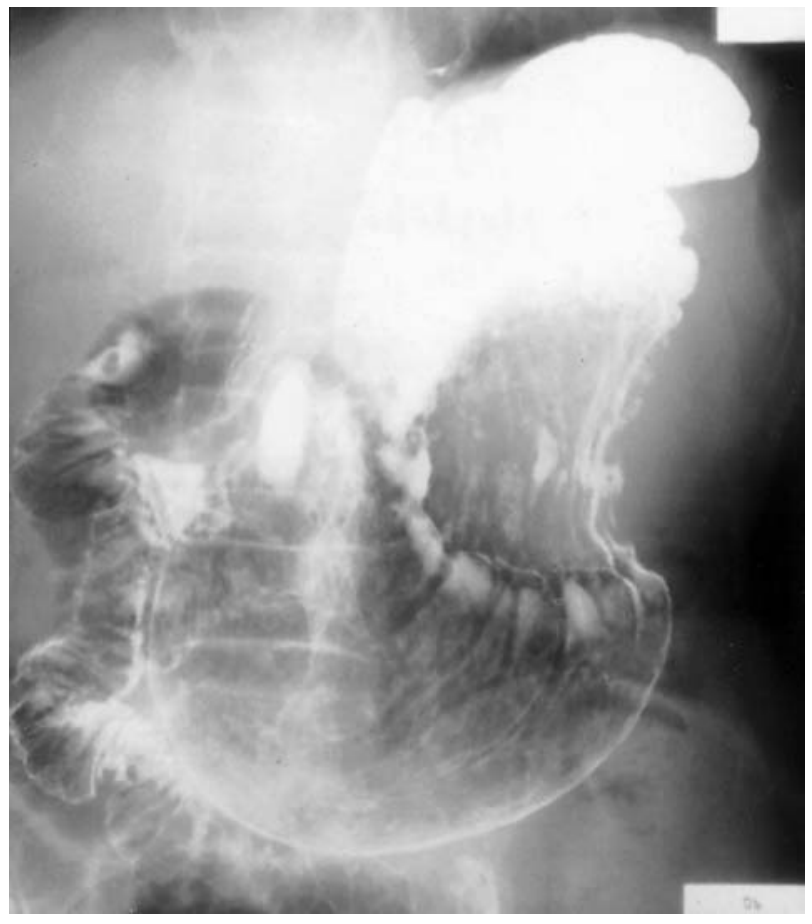

Fig. 1. Barium study shows a large submucosal tumor in the upper part of the stomach

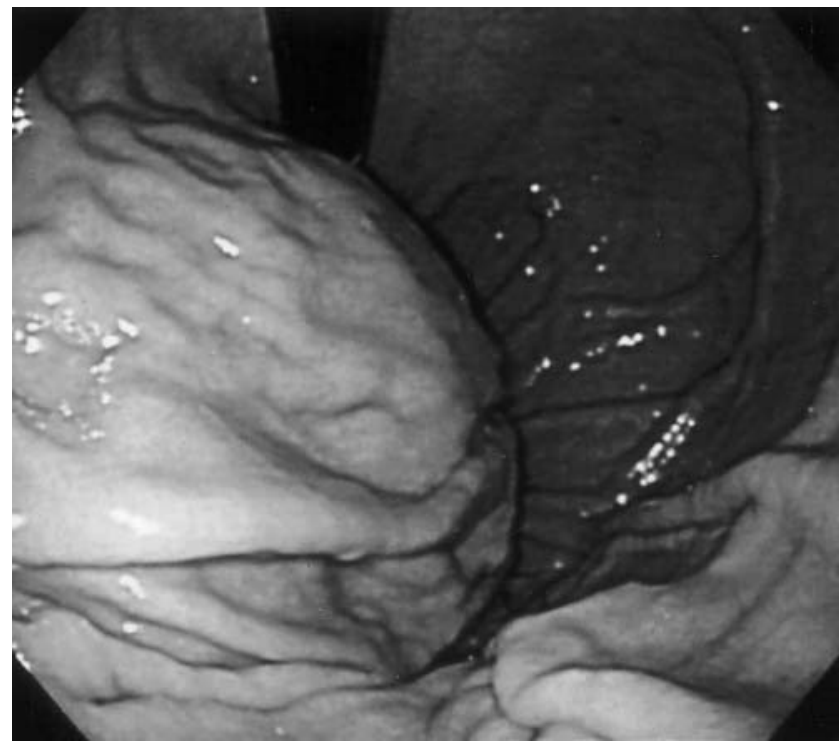

Fig. 2. Gastroscopic examination indicates a large submucosal tumor without ulceration

sigmoid colon cancer. We retrospectively examined these images and we were able to calculate the doubling time of the tumor accurately. By using NIH image software (ver 1.6.0, by Wayne Rasband; National Institutes of Health, Bethesda, MD, USA), the tumor volume was measured as $63.2 \mathrm{~cm}^{3}$ in September 2000, and as
$558.4 \mathrm{~cm}^{3}$ in July 2001 (Fig. 3A,B). The doubling time of this tumor was calculated as 3.3 months, which suggested a high growth rate and malignancy.

In August 2001, laparotomy was conducted. A 10-cmsized hard tumor, showing extraluminal growth from the greater curvature of the middle body of the stomach, was covered by the greater omentum (Fig. 4A). Partial gastric resection was performed. The patient recovered uneventfully and was transferred to a nearby hospital 7 days after the surgery.

Macroscopically, the specimen was a $9.0 \times 8.5 \times 5.5$ $\mathrm{cm}$-sized solid tumor, and the cut surface showed partial necrotic change (Fig. 4B). We also measured the tumor volume after fixation, by an in-water sedimentation method. The tumor volume was $510 \mathrm{~cm}^{3}$, indicating the accurate determination of tumor volume with computed tomographic volumetry. Microscopically, the tumor consisted of spindle-shaped cells with oval nuclei, and there were 15 to 16 mitoses per 50 high-power fields (HPFs), which indicated its malignant nature (Fig. 5). In immunohistochemical studies, the tumor was positive with respect to c-kit, CD34 (Fig. 6A,B), and vimentin, but negative with respect to smooth muscle actin and S-100 protein. Concerning the growth rate, $25.5 \%$ of the tumor cells were positive for Ki-67 antigen (MIB-1) in the most active area (Fig. 7). The final pathological diagnosis was malignant GIST.

The patient was found to have hepatic metastasis 27 months after the surgery, and he received hepatic subsegmentectomy in December 2003.

\section{Discussion}

Until quite recently, mesenchymal tumors of the gastrointestinal tract had generally been diagnosed as leiomyoma or leiomyosarcoma, based on microscopic findings [18]. The concept of gastrointestinal stromal tumor (GIST) has been changing with immunohistochemical and molecular technical advances [1,2]. Recently, it has been widely accepted that GIST is a mesenchymal tumor which expresses c-kit oncoprotein or has a mutation in either the c-kit or the plateletderived growth factor receptor-alfa (PDGFRA) gene $[2,3]$.

Several studies have revealed various types of c-kit mutations, including mutations in the juxtamembrane domain (exon 11), extracellular domain (exon 9), and tyrosine kinase domain (exons 13 and 17) [4-9]. Some authors have reported a correlation between the c-kit mutations and poor prognosis in patients with GISTs $[4,5]$, but others have reported no such correlations $[8,9]$.

Because the definition of GIST has been changed within a short time, there has been uncertainty con- 

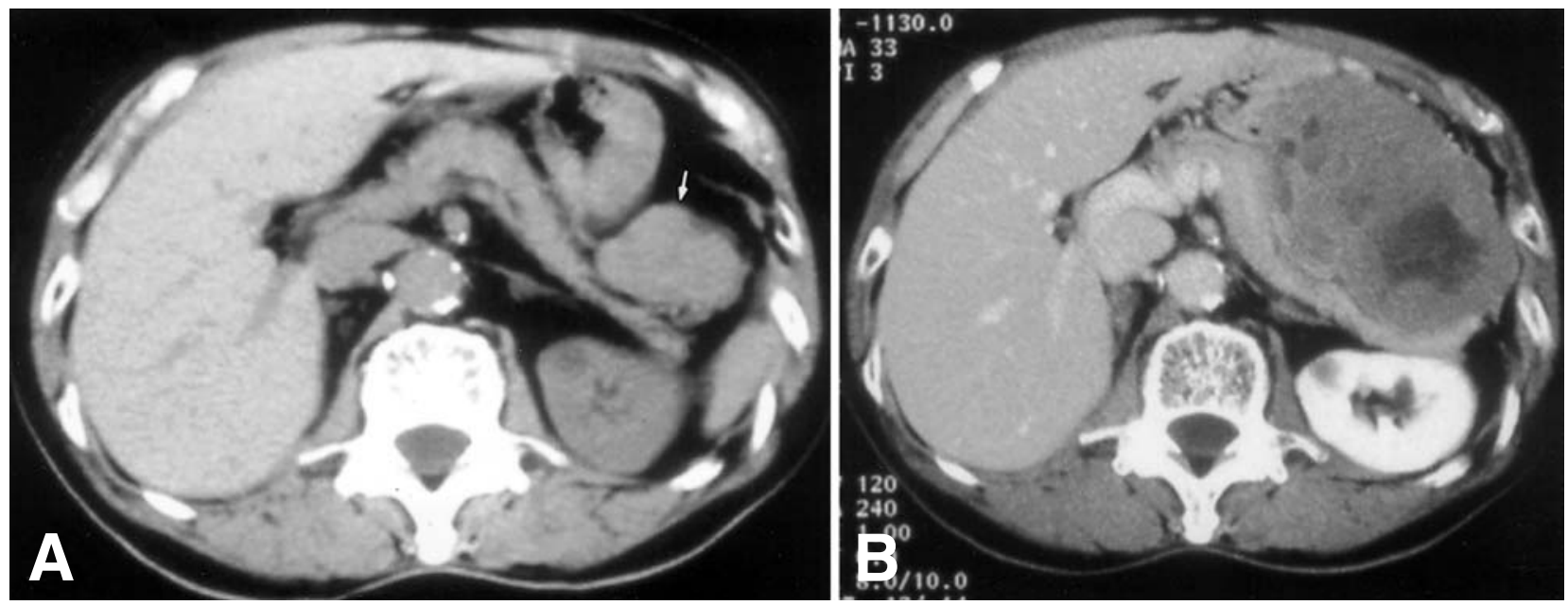

Fig. 3A,B. Computed tomography (CT) images. A Taken on September 13, 2000; B taken on July 18, 2001. The tumor had grown from $63.2 \mathrm{~cm}^{3}$ (arrow) to $558.4 \mathrm{~cm}^{3}$ in 308 days
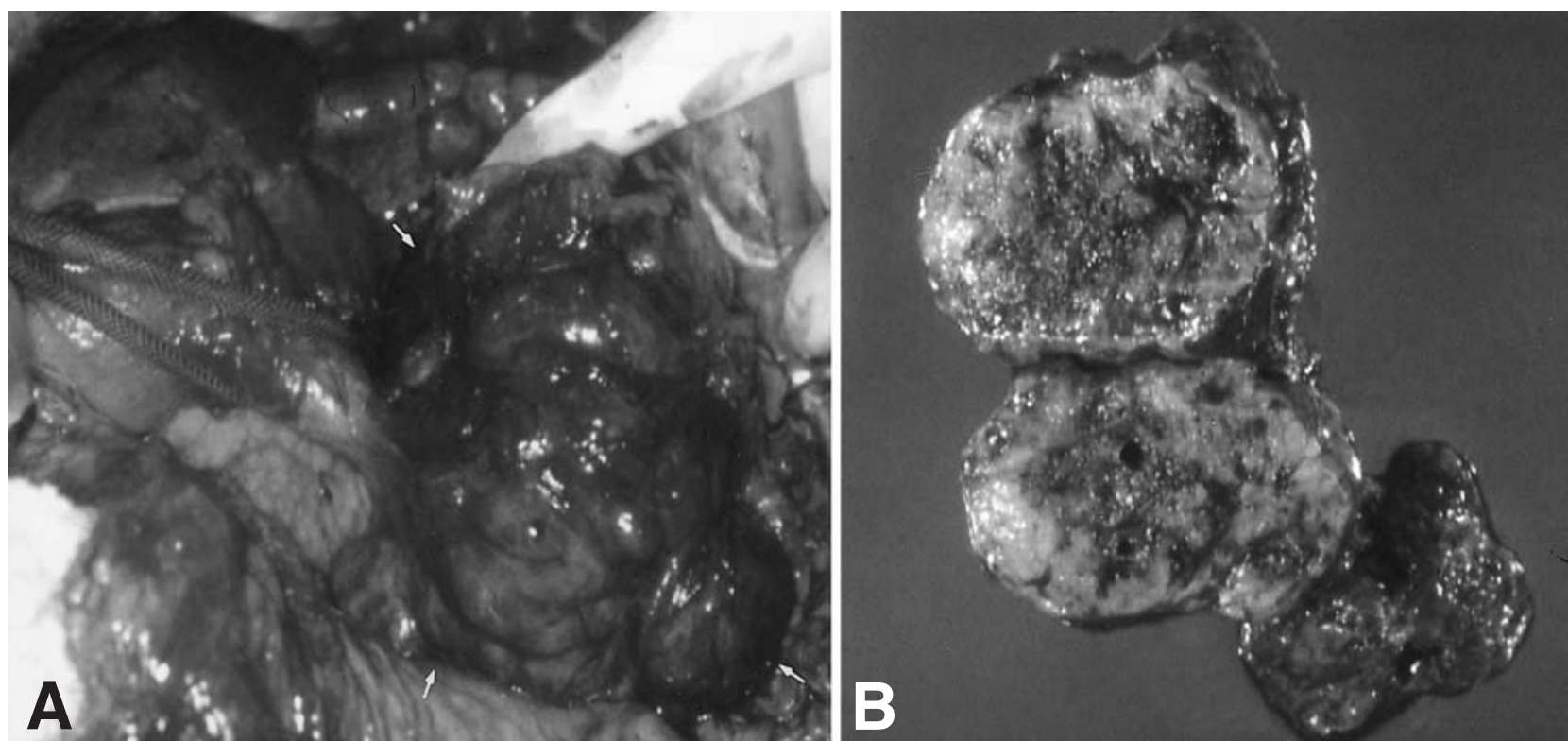

Fig. 4. A The tumor shows extraluminal growth (arrows), and is covered by the greater omentum. B Macroscopic view of the cut surface shows partial necrotic change

cerning GIST malignancy. Amin and Ma (Amin et al. [10] and Ma et al. [11]) proposed a standard of malignancy associated with the size of the tumor and the number of mitoses. They classified gastric stromal tumors into three groups: (1) benign $(<5 \mathrm{~cm}$ in size and $<$ five mitoses/50 HPFs), borderline $(>5 \mathrm{~cm}$ and $<$ five mitoses $/ 50 \mathrm{HPFs}$ ), and malignant ( $>$ five mitoses $/ 50$ HPFs). According to this classification, our case was classified in the malignant group. Iwashita et al. [12] reported that cellularity, type of growth, and clinical findings concerning the tumor (for example, necrosis, hemorrhage, and ulceration) were all useful in determining its malignancy. A proliferation index such as the MIB-1 index can be used for assessing prognosis [1317]. Carrillo et al [13] reported that a high MIB-1 index $(>22 \%$, in the most active area) was the most powerful predictor of poor survival. The highest MIB-1 index value for the present patient was $25.5 \%$, suggesting the malignant nature of the tumor. Rudolph et al. [14], Miettinen et al. [15], Panizo-Santos et al.[16], and Hasegawa et al. [17] noted that a mean proliferation index of more than $10 \%$ was highly predictive of a poor 

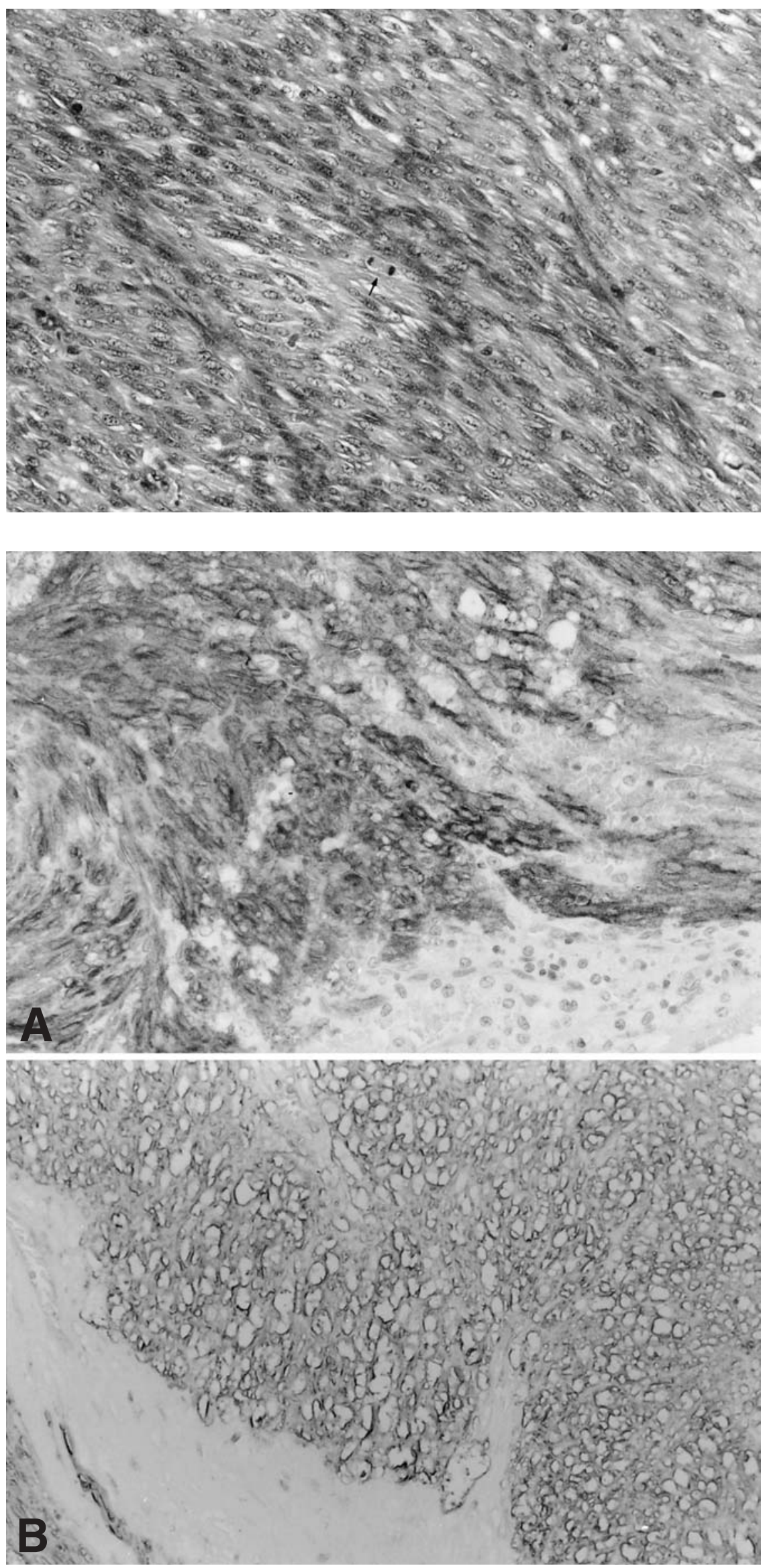

Fig. 5. Microscopically, the tumor consisted of spindle-shaped cells with oval nuclei, and some mitoses were observed (arrow). H\&E, $\times 200$
Fig. 6A,B. The tumor cells were immunoreactive for $\mathbf{A}$ c-kit, and $\mathbf{B}$ CD34. A $\times 200 ; \mathbf{B} \times 200$ 


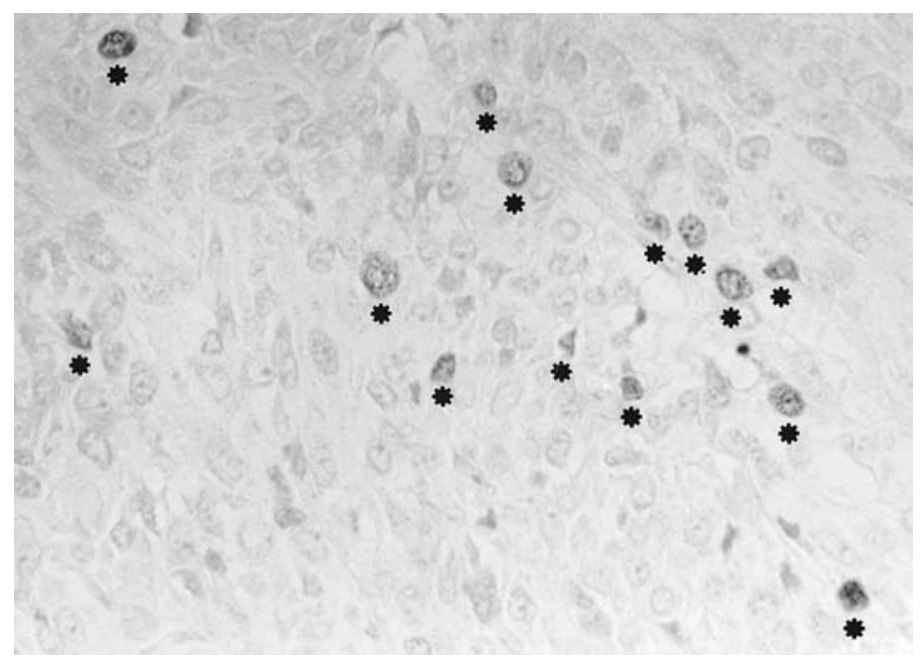

Fig. 7. In the most active area, $25.5 \%$ of tumor cells were positive for Ki-67 antigen (MIB-1) (asterisks). ×300

Table 1. Tumor doubling times of gastric mesenchymal tumors

\begin{tabular}{lll}
\hline Author (year) & $\begin{array}{c}\text { Doubling time of } \\
\text { leiomyoma } \\
\text { (GIST) }\end{array}$ & \multicolumn{1}{c}{$\begin{array}{c}\text { Doubling time of } \\
\text { leiomyosarcoma } \\
\text { (malignant GIST) }\end{array}$} \\
\hline Peck [19] (1968) & NR & 4.8 Months \\
Shimamoto [20] (1991) & $44.9(20.3-103.0)$ Months & $7.9(0.8-18.1)$ Months \\
Hamada [21] (1995) & $36.0(8.1-95.1)$ Months & $7.7(4.2-12.6)$ Months \\
Kimata [22] (2000) & 11.3 Months & 1.3 Months \\
Our patient & NR & 3.3 Months \\
\hline
\end{tabular}

Values in parentheses are ranges

NR, Not reported

prognosis. Past research concerning leiomyoma/leiomyosarcoma has also been useful, because many tumors of smooth-muscle origin would be diagnosed as GISTs in a narrow sense today.

There have been only a few reports concerning the doubling time of gastric submucosal tumors, and the diagnostic criteria could be different for different authors (Table 1) [19-22]. In 1968, Peck [19] reported that the doubling time in a case of leiomyosarcoma was 4.8 months. After that, Shimamoto et al. [20] calculated the mean doubling time as 44.9 months (range, 20.3-103.0 months) for leiomyoma, and 7.9 months (range, 0.818.1 months) for leiomyosarcoma. They advocated that surgical procedures should be implemented in cases that showed a doubling time of less than 20 months. Hamada et al. [21] estimated the mean doubling times as 36.0 months (range, 8.1-95.1 months) for leiomyoma and 7.7 months (range, 4.2-12.6 months) for leiomyosarcoma, respectively. Recently, Kimata et al. [22] reported that the doubling times in two cases of gastric GIST were 11.3 and 1.3 months.

In previous studies, the doubling time was calculated on the basis of barium meal results, assuming that the tumor was spherical. In the present patient, the tumor volume and the doubling time were calculated by computed tomographic volumetry, and the accuracy of the method was shown by comparison with the resected specimen. The calculated results showing a 3.3-month doubling time, indicated a high growth rate, and hence the tumor was categorized as malignant (Table 1). Actually, this patient was found to have hepatic metastasis 27 months after surgery, and he received a hepatic subsegmentectomy.

To date, there have been no clear standards for the preoperative assessment of GIST malignancy. Routine endoscopic biopsy samples rarely contain tumor components. Endoscopic bore biopsy and needle biopsy under endoscopic ultrasound seem to be useful for tumor assessment, but there is some risk of bleeding. Aspiration biopsy under extracorporeal ultrasound may induce peritoneal dissemination of malignant cells. We usually use clinical indices such as tumor diameter, tumor site, hemorrhage, and ulceration in assessing GIST malignancy. Many GISTs do not have clinical symptoms, and are often incidentally detected in the course of mass screenings or follow-up examinations for 
other diseases. If the GIST doubling time can be measured preoperatively, assessment of the tumor nature will be more reliable. The recent development of multidetector-row CT (MDCT) technology has brought about excellent three-dimensional (3D) imaging and analysis without difficulty. Computed tomographic volumetry is useful for accurately calculating doubling time, and may contribute to follow-up studies in patients with small $(<5 \mathrm{~cm}$ in size $)$ or irregularly shaped gastric submucosal tumors, as well as contributing to decisions regarding surgical intervention. MDCT may become the main modality for the follow up of gastric submucosal tumor.

In conclusion, we presented a case of gastric GIST. The doubling time of this tumor was accurately calculated by using computed tomographic volumetry. The calculated doubling time was 3.3 months, which indicated a high growth rate and malignancy. Partial gastric resection was performed, and the histological examination confirmed the preoperative assessment done by computed tomographic volumetry. Hepatic metastasis was found 27 months after surgery.

\section{References}

1. Rosai J. Stromal tumors. In: Rosai J, editor. Ackerman's surgical pathology. 8th ed. St. Louis: Mosby; 1996. pp. 645-7, 691-3.

2. Hirota S, Isozaki K, Moriyama $\mathrm{Y}$, Hashimoto $\mathrm{K}$, Nishida T, Ishiguro S, et al. Gain-of-function mutations of c-kit in human gastrointestinal stromal tumors. Science 1998;279:577-80.

3. Heinrich MC, Corless CL, Duensing A, McGreevey L, Chen CJ, Joseph N, et al. PDGFRA activating mutations in gastrointestinal stromal tumors. Science 2003;299:708-10.

4. Lasota J, Janinsky M, Sarlomo-Rikala M, Miettinen M. Mutations in exon 11 of c-kit occur preferentially in malignant versus benign gastrointestinal stromal tumors and do not occur in leiomyomas or leiomyosarcomas. Am J Pathol 1999;154:53-60.

5. Taniguchi $\mathrm{M}$, Nishida $\mathrm{T}$, Hirota $\mathrm{S}$, Isozaki $\mathrm{K}$, Ito Toshinori, Nomura T, et al. Effect of c-kit mutation on prognosis of gastrointestinal stromal tumors. Cancer Res 1999;59:4297-300.

6. Lasota J, Wozniak A, Sarlomo-Rikala M, Rys J, Kordek R, Nassar A, et al. Mutations in exon 9 and 13 of kit gene are rare events in gastrointestinal stromal tumors: a study of 200 cases. Am J Pathol 2000;157:1091-5.

7. Lux ML, Rubin BP, Biase TL, Chen CJ, Maclure T, Demetri G, et al. Kit extracellular and kinase domain mutations in gastrointestinal stromal tumors. Am J Pathol 2000;156:791-5.
8. Rubin BP, Singer S, Tsao C, Duensing A, Lux ML, Ruiz R, et al. Kit activation is a ubiquitous feature of gastrointestinal stromal tumors. Cancer Res 2001;61:8118-21.

9. Corless CL, McGreevey L, Haley A, Town A, Heinrich MC. Kit mutations are common in incidental gastrointestinal stromal tumors 1 centimeter or less in size. Am J Pathol 2002;160:1567-72.

10. Amin MB, Ma CK, Linden MD, Kubus JJ, Zarbo RJ. Prognostic value of proliferating cell nuclear antigen index in gastric stromal tumors: correlation with mitotic count and clinical outcome. Am J Clin Pathol 1993;100:428-32.

11. Ma CK, Amin MB, Kintanar E, Linden ND, Zabro RJ. Immunohistological characterization of gastrointestinal stromal tumors: a study of 82 cases compared with 11 cases of leiomyomas. Mod Pathol 1993;6:139-44.

12. Iwashita A, Ohshige Y, Haraoka S, Oseki M, Yao T, Kikuchi A, et al. Clinical pathology of gastrointestinal stromal tumor (GIST): with special reference to change of the concept of gastrointestinal mesenchymal tumor (GIMT) and the definition and organ specificity of GIST (in Japanese with English abstract). Stomach and Intestine 2001;36:1113-27.

13. Carrillo R, Candia A, Rodriguez-Peralto JL, Caz V. Prognostic significance of DNA ploidy and proliferating index (MIB-1 index) in gastrointestinal stromal tumors. Hum Pathol 1997;28:160-5.

14. Rudolph P, Gloeckner K, Parwaresch R, Harms D, Schmidt D. Immunophenotype, proliferation, DNA ploidy, and biological behavior of gastrointestinal stromal tumors: a multivariate clinicopathologic study. Hum Pathol 1998;29:791-800.

15. Miettinen M, Sarlomo-Rikala M, Lasota J. Gastrointestinal stromal tumors: recent advances in understanding of their biology. Hum Pathol 1999;30:1213-20.

16. Panizo-Santos A, Sola I, Vega F, Alava E, Lozano MD, Idoate MA, et al. Predicting metastatic risk of gastrointestinal stromal tumors: role of cell proliferation and cell cycle regulatory proteins. Int J Surg Pathol 2000;8:133-44.

17. Hasegawa T, Matsuno Y, Shimoda T, Hirohashi S. Gastrointestinal stromal tumor: consistent CD117 immunostaining for diagnosis, and prognostic classification based on tumor size and MIB-1 grade. Hum Pathol 2002;33:669-76.

18. Stout AP. Bizarre smooth muscle tumors of the stomach. Cancer 1962;15:400-9.

19. Peck HM. Gastric leiomyosarcoma with remarkable progress over a 2-year period. J Mt Sinai Hosp NY 1968;35:199-206.

20. Shimamoto T, Haruma K, Tokumo K, Suzuki T, Sumii K, Kajiyama G, et al. Growth rates of smooth muscle tumors of the stomach (in Japanese with English abstract). Jpn J Cancer Clin 1991;37:733-9.

21. Hamada S, Ohara S, Sekine H, Nakayama Y, Sugiyama K, Nishimura $\mathrm{T}$, et al. A differential diagnosis of gastric myogenic tumors from the clinical course: from a study of course-observed cases (in Japanese with English abstract). Stomach and Intestine 1995;30:1141-50.

22. Kimata M, Kubota T, Otani Y, Ohgami M, Ishikawa Y, Yokoyama T, et al. Gastrointestinal stromal tumors treated by laparoscopic surgery: report of three cases. Surg Today 2000;30: $177-80$. 